IMECE2002-32974

\title{
NATURAL CONVECTION MEASUREMENTS FOR A CONCENTRIC SPHERICAL ENCLOSURE
}

\author{
Peter M. Teertstra, M. Michael Yovanovich and J. Richard Culham \\ Microelectronics Heat Transfer Laboratory \\ Department of Mechanical Engineering \\ University of Waterloo \\ Waterloo, Ontario, Canada N2L 3G1 \\ Email: pmt@mhtlab.uwaterloo.ca \\ mmyov@mhtlab.uwaterloo.ca \\ rix@mhtlab.uwaterloo.ca
}

\begin{abstract}
An experimental test program is described for the measurement of natural convection for an isothermal, heated sphere centrally located in an isothermal, cooled spherical enclosure. A transient test method is used in a reduced pressure environment to provide data for a wide range of Rayleigh number, from the limiting case of laminar boundary layer convection to the diffusive limit. Tests are performed using a fixed outer diameter for four different inner sphere diameters, resulting in diameter ratios in the range $1.5 \leq d_{o} / d_{i} \leq 4.8$. The data are in excellent agreement with the exact solution for the conductive limit and are shown to be bounded by a model for the isolated, isothermal sphere.
\end{abstract}

\section{NOMENCLATURE}

A surface area; $m^{2}$

$a, b, c$ radiation correlation coefficients

$C$ coefficient

$c_{p} \quad$ specific heat capacity; $\mathrm{J} / \mathrm{kgK}$

$d$ diameter; $m$

$F(P r)$ Prandtl number function

$G_{\mathcal{L}}$ body gravity function

$g$ gravitation acceleration; $\mathrm{m} / \mathrm{s}^{2}$

$h$ convective heat transfer coefficient; $W / m^{2} K$

$k$ thermal conductivity; $W / m K$

$K n \quad$ Knudsen number, $\equiv \lambda / L$
I heater current; $A$

$L_{e} \quad$ effective fin length; $m$

$\mathcal{L}$ general characteristic length; $m$

$m$ mass; $k g$

$N u_{\mathcal{L}} \quad$ Nusselt number, $\equiv Q \mathcal{L} /\left[k A_{i} \Delta T\right]$

$P$ perimeter; $m$

$p$ pressure; $\mathrm{Pa}$

$P r$ Prandtl number

$Q$ heat flow rate; $W$

$R$ thermal resistance; $K / W$

$\mathcal{R}$ gas constant for air @STP; $287 \mathrm{~J} / \mathrm{kgK}$

$R a_{\mathcal{L}} \quad$ Rayleigh number, $\equiv g \beta \Delta T \mathcal{L}^{3} /(v \alpha)$

$S_{\mathcal{L}}^{\star} \quad$ conduction shape factor, $\equiv Q \mathcal{L} /\left[k A_{i} \Delta T\right]$

$t$ time; $s$

$T$ temperature; ${ }^{\circ} \mathrm{C}$

$\bar{T}$ average temperature; ${ }^{\circ} \mathrm{C}$

$\Delta T$ temperature difference, $\equiv T_{i}-T_{o} ;{ }^{o} C$

$V$ heater voltage; $V$

$Z$ compressibility factor

Greek Symbols

$\alpha$ thermal diffusivity; $\mathrm{m}^{2} / \mathrm{s}$

$\beta$ thermal expansion coefficient; $1 / K$

$\delta \quad$ gap thickness, $\equiv\left(d_{o}-d_{i}\right) / 2 ; m$

$\phi$ dimensionless temperature excess

$\lambda$ mean free path; $m$

$\Lambda$ thermocouple wire dimensions; $m$ 
$\mu \quad$ dynamic viscosity; $N \cdot s / m^{2}$

$v$ kinematic viscosity; $\mathrm{m}^{2} / \mathrm{s}$

$\rho$ mass density; $\mathrm{kg} / \mathrm{m}^{3}$

$\sigma \quad$ Stefan-Boltzmann constant; $W / m^{2} K^{4}$

$\tau$ time constant, $\equiv m c_{p} R ; s$

$\theta$ temperature excess

$\xi$ fin parameter; $1 / m$

$\zeta$ radiation parameter, $\equiv \sigma\left(T_{i}^{4}-T_{o}^{4}\right) ; W / m^{2}$

\section{Subscripts \\ $b$ bulk fluid \\ $i$ inner body \\ $o$ outer body \\ cond conduction \\ conv convection \\ rad radiation losses \\ eff effective \\ tot total \\ $\ell$ conduction losses \\ $w$ wire conductor \\ ins wire insulation}

\section{Superscripts}

* dimensionless quantity

\section{INTRODUCTION}

The problem of natural convection in the enclosed region formed between an isothermal heated body and its surrounding, isothermal cooled enclosure is currently of some interest to designers of microelectronics equipment. In an effort to protect electronics from environmental contaminants such as dust or moisture, circuits are often housed in sealed enclosures, especially in outside plant applications. The ability to model natural convection heat transfer within these sealed enclosures would be of great benefit, providing quick and easy-to-use design tools for preliminary design tasks such as parametric studies and trade-off analysis.

Research is currently underway to develop analytically based models to predict convective heat transfer in these systems. Of particular importance to the model development process is the enclosure formed between isothermal concentric spheres, the most fundamental type of doubly-connected enclosure. It is anticipated that the lessons learned during the development of a natural convection model for the concentric spheres will be directly applicable to more complex enclosure geometries.

One of the most important elements in the development of analytical models is the availability of experimental data over the full range of the independent parameters. Accurate data are vital in order to reveal trends, such as limiting cases or transition behavior, and for the validation of the completed models. The current literature contains only a limited set of experimental data for

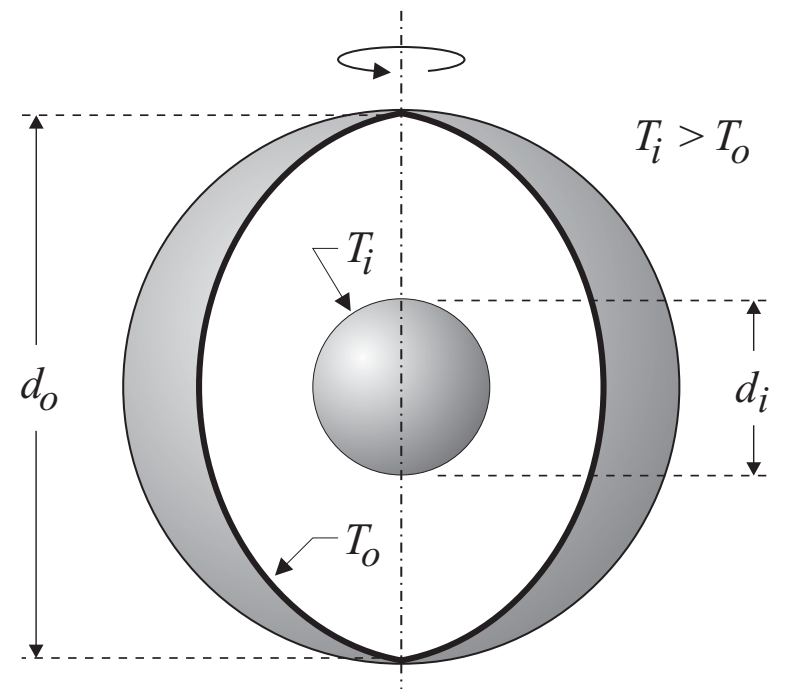

Figure 1. SCHEMATIC OF CONCENTRIC SPHERICAL ENCLOSURE.

the isothermal concentric sphere problem. Bishop et al. (1966) performed air measurements at atmospheric pressure for a single outer sphere diameter and four inner sphere diameters, leading to diameter ratios of $d_{o} / d_{i}=1.25,1.67,2.0$ and 2.5. The data are limited to a narrow range of Rayleigh number, approximately 1 decade, at the high Rayleigh number, boundary layer flow limit. Scanlan et al. (1970) performed measurements for water and silicon oil-filled spherical enclosures, with $4.7 \leq \operatorname{Pr} \leq 4148$ for five diameters ratios ranging from $d_{o} / d_{i}=1.09$ to $2 . \overline{8} 1$. Again, the data are limited to large values of $R a_{d_{i}}$, and the data are presented without any means to differentiate between the different diameter ratios. There are no experimental data in the current literature for the isothermal concentric spherical enclosure valid for the full range of Rayleigh number that includes the transition from convection to conduction-dominated heat transfer.

The objective of the current study is to perform measurements of natural convection heat transfer for isothermal concentric spheres for the full range of Rayleigh number, from the laminar boundary layer flow limit to the conductive limit. The procedure for performing the measurements will be developed, the test apparatus will be described, and data for four different diameter ratios will be presented.

\section{PROBLEM DEFINITION}

The problem of interest involves convective heat transfer from a sphere, diameter $d_{i}$, to a concentric spherical shell with inner diameter $d_{o}$, as shown in Figure 1. Isothermal boundary conditions exist at both the inner and outer boundaries, as follows: 


$$
\begin{array}{ll}
\text { Inner Boundary } & T=T_{i} \\
\text { Outer Boundary } & T=T_{o}
\end{array}
$$

where $T_{i}>T_{o}$. The total heat transfer rate through the enclosed region is determined at the inner boundary by:

$$
Q=\iint_{A_{i}}-k \frac{\partial \theta}{\partial n_{A_{i}}} d A, \theta=T(\vec{r})-T_{b}
$$

where $T(\vec{r})$ is the temperature distribution adjacent to the inner boundary along an outward-facing normal, and $T_{b}$ is the bulk fluid temperature in the enclosure. Assuming constant fluid properties and non-dimensionalizing yields the dimensionless total heat transfer rate:

$$
Q_{\mathcal{L}}^{\star}=\frac{Q \mathcal{L}}{k A_{i}\left(T_{i}-T_{o}\right)}=\frac{\mathcal{L}}{A_{i}} \iint_{A_{i}}-\frac{\partial \phi}{\partial n_{A_{i}}} d A
$$

where $\mathcal{L}$ is a general scale length and the dimensionless temperature excess $\phi$ is defined as:

$$
\phi=\frac{T(\vec{r})-T_{b}}{T_{i}-T_{o}}
$$

The average heat transfer coefficient for the enclosure $h$ is defined based on the average heat flux at the inner boundary and the overall temperature difference:

$$
h=\frac{\left(Q / A_{i}\right)}{\Delta T}
$$

where $\Delta T=T_{i}-T_{o}$. Non-dimensionalizing $h$ using the general scale length $\mathcal{L}$ gives the area-mean Nusselt number, which can be shown to be equivalent to the dimensionless heat transfer rate:

$$
N u_{\mathcal{L}}=\frac{h \mathcal{L}}{k}=\frac{Q \mathcal{L}}{k A_{i} \Delta T}=Q_{\mathcal{L}}^{\star}
$$

The Rayleigh number is defined using the same parameters:

$$
R a_{\mathcal{L}}=\frac{g \beta \Delta T \mathcal{L}^{3}}{v \alpha}
$$

There exists several methods whereby the Rayleigh number can be varied during experimental testing. Changes to the temperature difference, $\Delta T$, result in only small variations in $R a_{\mathcal{L}}$, typically less than 1 decade. Varying the dimensions of the body requires fabrication and testing of a number of specimens of different sizes. The best method for providing a large variation in the Rayleigh number for natural convection is through variation of the fluid properties by a change of the gas pressure, as described by Saunders (1936) and Hollands (1988). Varying $R a_{\perp}$ by applying a partial vacuum to the test environment allows the use of a single test specimen operating over a small temperature difference to easily span four or more decades of Rayleigh number.

Modeling the air in the enclosure as an ideal gas at bulk temperature $T_{b}$ gives the following expression for the density:

$$
\rho=\frac{p}{\mathcal{R} T_{b} Z}
$$

where $\mathcal{R}$ is the gas constant for air and $Z$ is the compressibility factor for air. Substituting into Eq. (6) gives a new definition for Rayleigh number as a function of $p$ :

$$
R a_{\mathcal{L}}=\frac{g \beta \Delta T \mathcal{L}^{3} p^{2} c_{p}}{\mathcal{R}^{2} T_{b}^{2} k \mu Z^{2}}
$$

where the fluid properties, $\beta, c_{p}, k$ and $\mu$, are constant with respect to pressure and are evaluated at the bulk temperature, $T_{b}$. The compressibility, $Z$, is a function of both the bulk fluid temperature and pressure.

For values of $R a_{\mathcal{L}}$ less than some critical value, the heat transfer in the enclosure is conduction-dominated and independent of Rayleigh number. In these cases, the dimensionless heat transfer rate is equivalent to the dimensionless conduction shape factor, $Q_{\mathcal{L}}^{\star}=S_{\mathcal{L}}^{\star}$, which is defined by Yovanovich as (1998):

$$
S_{\mathcal{L}}^{\star}=\frac{\mathcal{L}}{A_{i}} \iint_{A_{i}}-\frac{\partial \phi}{\partial n_{A_{i}}} d A
$$

The conduction shape factor is related to the thermal resistance by:

$$
S_{\mathcal{L}}^{\star}=\frac{1}{k \mathcal{L} R}
$$

Using the exact solution for the thermal resistance of a concentric spherical shell (Incropera and DeWitt, 1996), the conduction shape factor is:

$$
S_{\mathcal{L}}^{\star}=\frac{2 \mathcal{L}}{d_{i}\left(1-\frac{d_{i}}{d_{o}}\right)}
$$

Since the problem of interest involves only spherical body shapes and the size of the inner sphere is the only variable geometric parameter, the diameter of the inner sphere is selected 


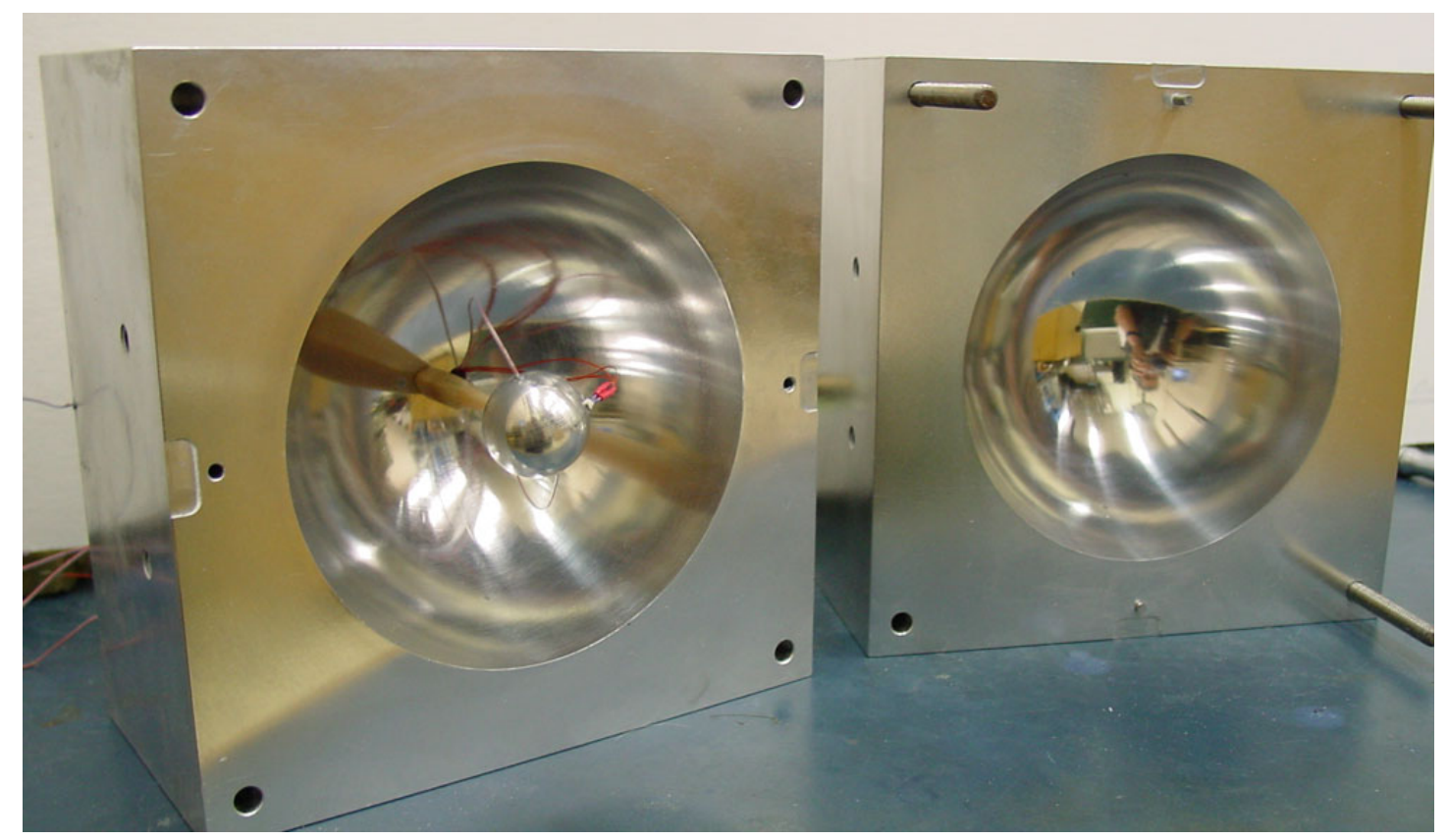

Figure 2. SPHERICAL ENCLOSURE WITH $25 \mathrm{~mm}$ DIAMETER SPHERE.

as the scale length for all dimensionless parameters, such that $\mathcal{L}=d_{i}$.

\section{EXPERIMENTAL APPARATUS}

In order to perform the required measurements, an experimental apparatus was created consisting of a single, spherical enclosure and a series of inner spheres of various diameters. The outer spherical enclosure was constructed of two aluminum blocks with hemispherical cavities machined into one side, as shown in Figure 2, so as to form a spherical shell when joined together. Aluminum 6061 was used due to its high value of thermal conductivity to provide a near-isothermal boundary condition, and the hemispherical surfaces were polished to minimize radiation heat transfer.

The size of the enclosure was selected based on two main criteria. First, because many of the tests were to be performed at reduced pressures, it was necessary that the maximum dimensions of the apparatus not exceed the space available within the available vacuum chamber. Second, in order to avoid rarefaction effects the gap spacing between the inner and outer boundaries, $\delta$, had to be much larger than the mean free path of gas, $\lambda$, as defined by the Knudson number:

$$
K n=\frac{\lambda}{\delta}
$$

The mean free path of air as a function of pressure and temperature can be determined by (Kennard, 1938):

$$
\lambda=6.4 \times 10^{-8}\left(\frac{1}{p(\mathrm{~atm})}\right)\left(\frac{T(K)}{288}\right)(\mathrm{m})
$$

Ensuring that $K n<0.01$ for the full range of pressures and temperatures anticipated in the experimental program provides a lower limit for the outer sphere dimensions. Based on these two criteria, the dimensions of the outer enclosure were chosen such that its diameter was $d_{o}=120 \mathrm{~mm}$.

In order to provide data for a wide range of diameter ratios, four different spherical inner bodies were machined from 6061 aluminum. The diameters of the spheres and the resulting diameter ratios are given in Table 1. Each sphere was suspended at the center of the enclosure using a $4-6 \mathrm{~mm}$ diameter threaded phenolic rod turned into tapped holes on both the inner and outer enclosure walls, as shown in Figure 3. All wiring to the inner sphere was connected through a single, $6 \mathrm{~mm}$ diameter hole at the top of the enclosure.

All temperature measurements were performed using T-type copper-constantan thermocouples affixed at the surfaces of the inner and outer spheres in shallow, small diameter holes using aluminum-filled epoxy. The temperature at the outer surface of the enclosure was measured using six 30 AWG $(0.254 \mathrm{~mm})$ thermocouple wires distributed at the top, bottom and midplane, while the two thermocouples at the top and bottom of the inner 
Table 1. ENCLOSURE DIMENSIONS.

\begin{tabular}{rcc}
\hline$d_{o}(\mathrm{~mm})$ & $d_{i}(\mathrm{~mm})$ & $d_{o} / d_{i}$ \\
\hline 120.0 & 80.0 & 1.5 \\
120.0 & 60.0 & 2.0 \\
120.0 & 40.0 & 3.0 \\
120.0 & 25.0 & 4.8 \\
\hline
\end{tabular}

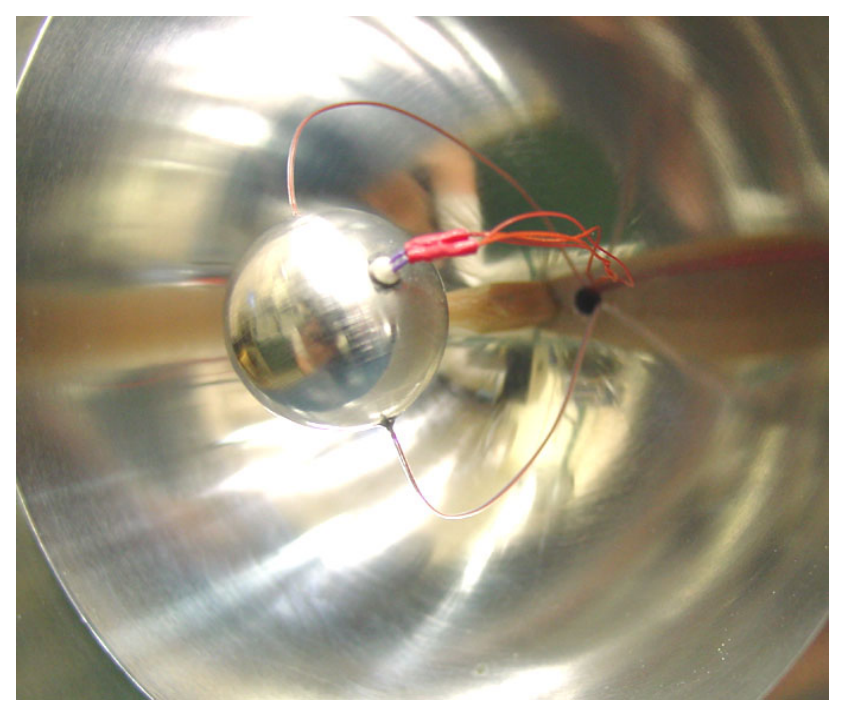

Figure 3. DETAIL OF $25 \mathrm{~mm}$ SPHERE MOUNTED IN ENCLOSURE

body used smaller diameter, 36 AWG $(0.127 \mathrm{~mm})$ wires to reduce conduction losses. All thermocouples measurements were based on an external reference junction that was maintained at $0 \pm 0.1^{\circ} \mathrm{C}$ by an ice point cell.

Each of the inner spheres were heated using an embedded DC-powered cartridge heater. Constantan wires were used for all connections rather than copper to reduce heat losses through the wires; 24 AWG $(0.508 \mathrm{~mm})$ to provide power to the heaters and 36 AWG to measure voltage. The current to the heater was measured using a calibrated shunt resistor.

The outer enclosure walls were cooled by six cold plates attached on the exterior surface of the blocks using thermallyconductive grease at the joints. Heat was removed from the system using a glycol-water mixture circulated through the cold plates by a constant temperature bath.

Once assembled, the enclosure test apparatus was placed in vacuum chamber, as shown in Figure 4, with feedthroughs available for the coolant, electrical and instrumentation connections. The vacuum chamber used in this work uses a dual-pump system; a mechanical roughing pump capable of providing reduced pres-

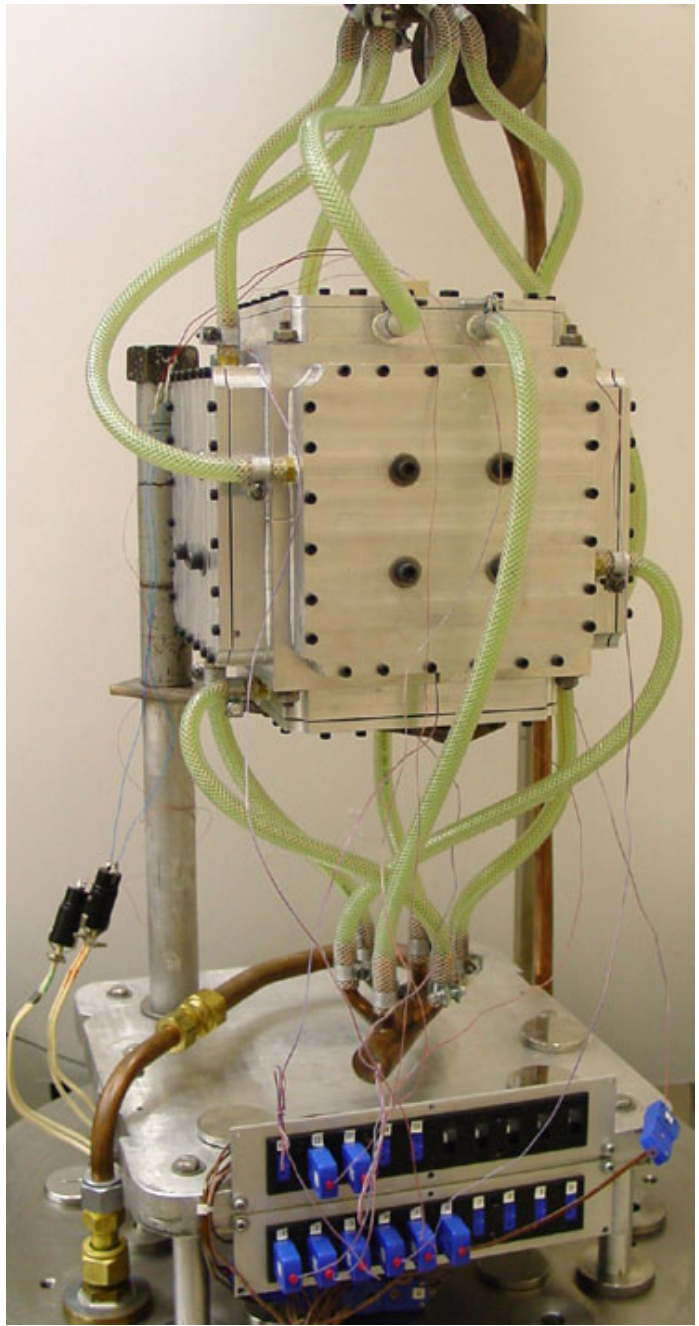

Figure 4. ENCLOSURE TEST APPARATUS IN VACUUM CHAMBER

sure test conditions suitable for the convection tests, and a diffusion pump for producing a totally evacuated environment for radiation heat transfer testing. The vacuum system also contains a high accuracy vacuum gauge suitable for absolute readings in the range $0.001-1 \mathrm{~atm}$.

Data acquisition and control of the experiment was performed using a Keithley 2700 data acquisition system and a Windows-based PC computer running Labview v.5.1 software.

\section{MEASUREMENT PROCEDURE}

The heat transfer rate due to convection through the enclosure, $Q$, can be determined based on an energy balance on the inner boundary:

$$
Q=Q_{t o t}-Q_{r a d}-Q_{\ell}
$$


where $Q_{\text {tot }}$ is the total heat transfer due to all modes, $Q_{\text {rad }}$ is the net radiative heat transfer between the inner and outer surfaces, and $Q_{\ell}$ are the accumulated conduction losses through the wires. In order to predict $Q$, a means is required whereby the total heat transfer rate can be measured, along with a method to quantify the losses due to radiation and conduction.

\section{Total Heat Transfer Rate}

The simplest method to determine the total heat transfer rate $Q_{t o t}$ is through a direct measurement of electrical energy dissipated by the heater during a steady-state test. A fixed voltage would be applied to the heater and the body temperatures would be monitored until sufficient time had elapsed such that the temperature change is less than some specified criteria. Then, the total heat transfer rate would be determined by:

$$
Q_{t o t}=V I
$$

where $V$ and $I$ are the heater voltage and current, respectively.

Hollands (1988) reports that, in the case of natural convection in gases, approximately 5 times the time constant, $5 \tau$, is required to achieve steady-state conditions, where the time constant is defined as:

$$
\tau=m c_{p} R
$$

Due to the relatively large values of the heat capacity, $m c_{p}$, and the average thermal resistance, $R$, for the proposed tests, especially those to be performed in a reduced pressure environment, steady-state testing becomes a prohibitively time-consuming option. Instead, the current study will implement the transient test method of Hollands (1988) that allows convective heat transfer measurements to be performed in a fraction of the time required for steady-state tests. This method is based on the assumption that, due to the slow rate of change of body temperature, a"quasisteady" condition exists where the convective heat transfer is virtually identical to the steady-state results at the same temperature.

The use of a transient test to measure steady-state convection in the enclosure can be validated by a comparison of the time constants for the inner body and the enclosed air layer for the worst case condition, the smallest sphere, $d_{i}=25 \mathrm{~mm}$, at atmospheric pressure. Using Eq. (15) and textbook values (Incropera and DeWitt, 1996) for the thermophysical properties $\rho$ and $c_{p}$, the time constant for the sphere is determined as a function of the film resistance at the inner boundary:

$$
\begin{aligned}
\tau_{i} & =\left(2770 \frac{\mathrm{kg}}{\mathrm{m}^{3}}\right)\left(8.18 \times 10^{-6} \mathrm{~m}^{3}\right)\left(875 \frac{\mathrm{J}}{\mathrm{kgK}}\right) \cdot R_{i} \\
& =19.8 \cdot R_{i}
\end{aligned}
$$

The time constant for the enclosed air layer, $\tau_{b}$, is determined using the same method:

$$
\begin{aligned}
\tau_{b} & =\left(1.1614 \frac{\mathrm{kg}}{\mathrm{m}^{3}}\right)\left(8.97 \times 10^{-4} \mathrm{~m}^{3}\right)\left(1007 \frac{\mathrm{J}}{\mathrm{kg} /, \mathrm{K}}\right) \cdot R_{o} \\
& =1.05 \cdot R_{o}
\end{aligned}
$$

where $R_{O}$ is the film resistance at the outer boundary. Assuming that the film resistances at the inner and outer surfaces are similar, $R_{i} \approx R_{o}$, the ratio of the time constants can be calculated:

$$
\frac{\tau_{i}}{\tau_{b}} \approx 19
$$

With a factor of 20 difference between the time constants for the worst case conditions, it is therefore reasonable to assume that the cooling rate of the inner body will control the heat transfer and that a "quasi-steady" condition exists in the enclosed fluid region.

In the transient test method, the body is heated to some initial, specified temperature while the temperature of the enclosure remains constant throughout the test. When the prescribed temperature difference is reached, the power to the heater is turned off and the transient response of the inner body is monitored. Measurements continue until $\Delta T$ falls below some minimum prescribed value. The total heat transfer rate at any time $t$ and corresponding temperature difference $\Delta T$ can be determined based on the transient data by:

$$
Q_{t o t}=-m c_{p} \frac{d T_{i}(t)}{d t}
$$

where the heat capacity of the inner body, $m c_{p}$, is determined empirically using a method described in the next section. The time derivative in Eq. (19) is approximated for distinct time intervals, $t_{n}$, using a least-squares method to predict the slope of sets of 101 average inner body temperature versus time data points.

$$
\left.\frac{d T}{d t}\right|_{t_{n}}=\operatorname{slope}\left(T_{i} \text { vs. } t\right) \text { for } 1 \leq j \leq 101
$$

Then, $Q_{t o t}$ can be calculated for time $t_{n}$, corresponding to the time value of the middle data point:

$$
t_{n}=t_{j=51}
$$

as shown in Figure 5. Corresponding values at time $t_{n}$ for the remaining parameters, $T_{i}, T_{o}, p$ and $V \cdot I$, are determined using an 


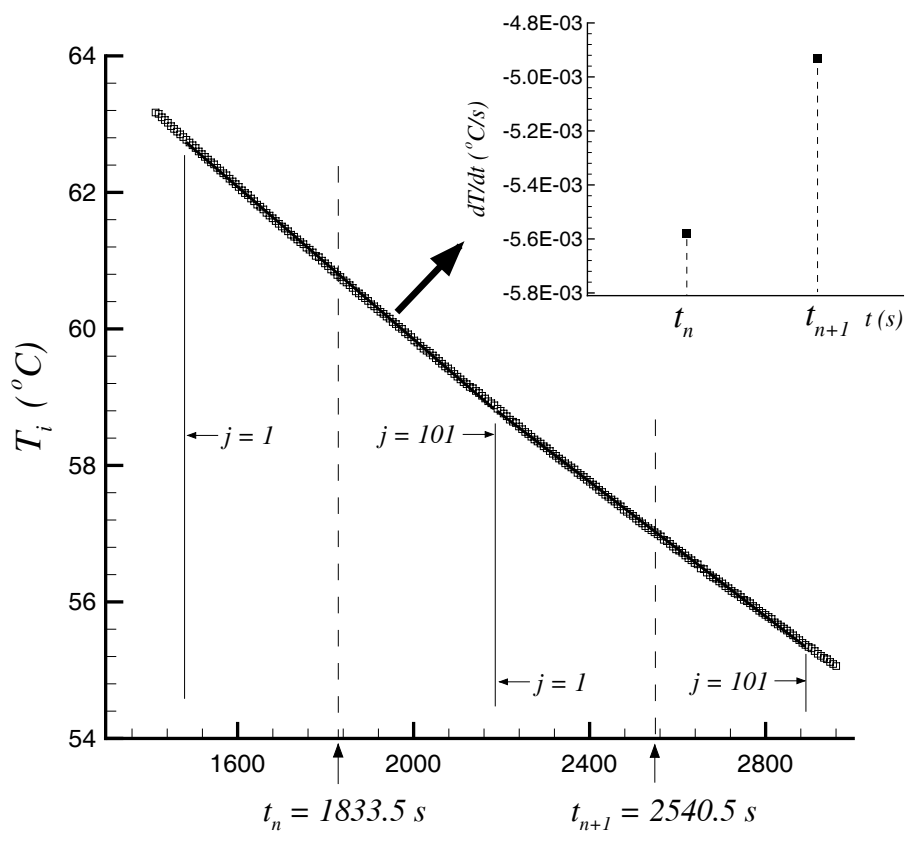

Figure 5. CALCULATION OF TIME DERIVATIVE OF TEMPERATURE, $d_{o} / d_{i}=1.5$.

arithmetic average of 9 values around the middle data point, as shown below and in Figure 6 for the example of the inner body temperature.

$$
T_{i, n}=\frac{1}{9} \sum_{j=47}^{9} T_{i, j}
$$

Using this transient test method and data reduction procedure, $Q_{t o t}$ is determined for a number of $\Delta T$ values between the start and end conditions, where the number of points depends on the heat capacity of the body, the convective conditions and the time step selected for the measurement.

\section{Heat Capacity and Radiative Losses}

The simplest method for determining the heat capacity of an isotropic body is to measure its mass and multiply by a tabulated value for specific heat capacity value from a handbook. However, in the case of the current study the inner spheres are not homogeneous but instead contain an embedded cartridge heater, a section of the phenolic mounting rod and thermocouples. The radiation heat transfer through the enclosure could also be modeled using available analytical techniques, but without precise values for the emissivity of the inner and outer boundaries, it is difficult to produce accurate results. Therefore, both the heat capacity and the

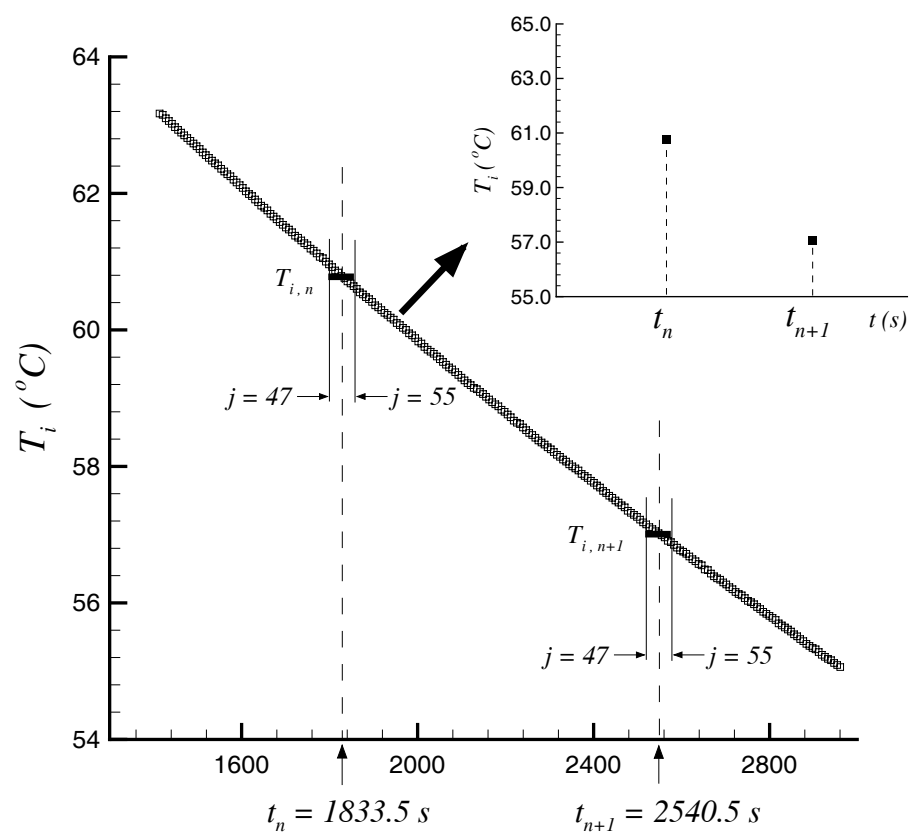

Figure 6. CALCULATION OF AVERAGE INNER BODY TEMPERATURE, $d_{o} / d_{i}=1.5$.

radiative heat transfer for each test case will be determined based on empirical data.

In order to provide an effective measure of both quantities, a two-stage test procedure is used. Starting at an initial condition $\Delta T \approx 0$, a heating test to a maximum value of $\Delta T$ is performed to determine the heat capacity, followed immediately by a cooling test back to a final $\Delta T$ value to measure radiative losses. For high vacuum conditions, such that $K n>100$, it can be assumed that gaseous convection and conduction are eliminated and heat transfer occurs by radiation alone. The energy balance for the inner body for any time $t$ is:

$$
m c_{p} \frac{d T_{i}}{d t}=V \cdot I-Q_{\mathrm{rad}}
$$

In the heating test the constant value of electrical power $V \cdot I$ provided to the heater can be assumed to be large enough such that non-linear effects can be neglected and $Q_{\text {rad }}$ can be assumed to be a linear function of the factor $\sigma\left(T_{i}^{4}-T_{o}^{4}\right)$. Then Eq. (22) becomes:

$$
\frac{d T_{i}}{d t}=\frac{V \cdot I}{m c_{p}}-\frac{C_{r a d}}{m c_{p}} \sigma\left(T_{i}^{4}-T_{o}^{4}\right)
$$

where $C_{\text {rad }}$ is assumed to be constant for each test body. Using a least squares method to determine the time gradient of the inner 


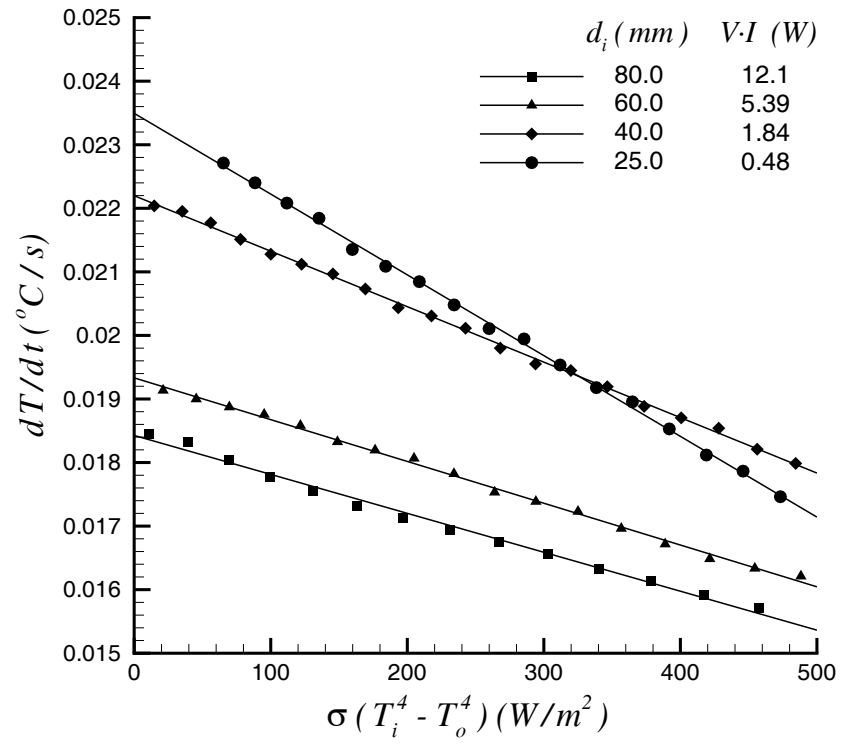

Figure 7. HEATING TEST DATA

Table 2. HEAT CAPACITY VALUES FOR INNER SPHERICAL BODIES

\begin{tabular}{cc}
\hline$d_{i}(m m)$ & $m c_{p}$ \\
\hline 80.0 & 653 \\
60.0 & 279 \\
40.0 & 82.9 \\
25.0 & 20.3 \\
\hline
\end{tabular}

body temperature, as described in the previous section, values of $d T / d t$ can be plotted versus the radiation parameter $\sigma\left(T_{i}^{4}-T_{o}^{4}\right)$, as shown in Figure 7. Based on Eq. (23) it can be seen that the y-intercept predicted by the linear fit of the data in Figure 7 can be used to calculate the heat capacity of the body. The empirical predictions for the heat capacity of each of the inner spheres tested are presented in Table 2.

Once the maximum $\Delta T$ value had been achieved, the heater was shut down, $V \cdot I=0$, reducing Eq. (22) to:

$$
Q_{\text {rad }}=-m c_{p} \frac{d T_{i}}{d t}
$$

where $d T / d t$ is determined using the least squares approximation described in the previous section. Figure 8 presents the measured values of $Q_{\text {rad }}$ versus the radiation parameter, $\sigma\left(T_{i}^{4}-T_{o}^{4}\right)$. As can be seen from Figure 8, the radiative heat transfer is a nonlinear function, due to conduction losses in the connecting rod
Table 3. RADIATION CORRELATION COEFFICIENTS

\begin{tabular}{cccc}
\hline$d_{i}(\mathrm{~mm})$ & $a \times 10^{10}$ & $b \times 10^{7}$ & $c \times 10^{4}$ \\
\hline 80.0 & 59.77 & -19.49 & 7.642 \\
60.0 & 7.855 & -1.562 & 5.255 \\
40.0 & 9.198 & -2.240 & 3.350 \\
25.0 & 1.932 & -0.750 & 2.147 \\
\hline
\end{tabular}

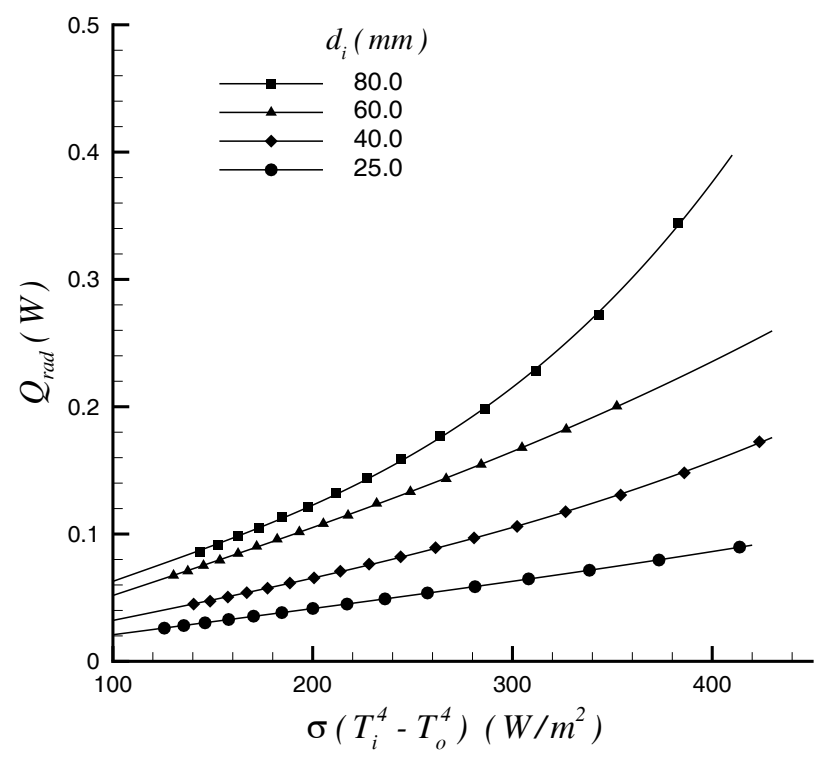

Figure 8. COOLING TEST DATA

and wires, and temperature dependence of the emissivity. In order to quantify these losses, an empirical relationship is developed for each inner body based on a third-order polynomial fit, as shown in Figure 8. The empirical relationship is:

$$
Q_{r a d}=a \zeta^{3}+b \zeta^{2}+c \zeta
$$

where $\zeta$ is the radiation parameter, $\sigma\left(T_{i}^{4}-T_{o}^{4}\right)$, and the correlation coefficients for each body are presented in Table 3 .

\section{Conduction Losses}

There are four potential sources of conductive heat loss from the inner body: the power wires, the voltage measurement leads, the thermocouples and the connecting rod. This analysis will consider losses by convection from the wires only; it is assumed that losses due to radiation from the wires and conduction losses 
through the connecting rod have been accounted for by the correlation of the radiation test data.

The heat loss from each individual wire connected to the inner body can be modeled as an infinitely long fin (Incropera and DeWitt, 1996)

$$
Q_{\ell}=\sqrt{h_{e f f} P_{w} k_{w} A_{w}}\left(T_{i}-T_{b}\right)
$$

where $P_{w}$ and $A_{w}$ are the perimeter and cross sectional area of the conductor, $k_{w}$ is the thermal conductivity of the conductor material and $T_{b}$ is the bulk fluid temperature. The effective heat transfer coefficient value, $h_{e f f}$, has been modified to include the conductive resistance of the insulation on the wire, as described by Sparrow (1976). By assuming a series combination of a conduction resistance through a circular annulus and the convective film resistance at the insulation surface, the effective heat transfer coefficient can be determined by:

$$
\begin{aligned}
h_{e f f} & =\frac{1}{\pi d_{w} L_{e}\left(R_{\text {cond }}+R_{\text {conv }}\right)} \\
& =\frac{1}{d_{w}\left[\frac{\ln \left(d_{\text {ins }} / d_{w}\right)}{2 k_{\text {ins }}}+\frac{1}{h d_{\text {ins }}}\right]}
\end{aligned}
$$

where $d_{i n s}$ and $k_{\text {ins }}$ are the diameter and thermal conductivity of the insulation and $L_{e}$ is an effective fin length. Convective heat transfer from the insulation is modeled as an infinitely long, horizontal circular cylinder (Raithby and Hollands, 1998):

$$
h=\frac{k}{d_{\text {ins }}} \frac{2}{\ln \left[1+\frac{2}{0.403 R a_{d_{\text {ins }}^{1 / 4}}^{1}}\right]}
$$

where the Rayleigh number is modified to include the gas pressure as described previously:

$$
R a_{d_{i n s}}=\frac{g \beta\left(\bar{T}_{w}-T_{b}\right) d_{i n s}^{3} p^{2} c_{p}}{R^{2} T_{b}^{2} k \mu Z^{2}}
$$

The bulk fluid temperature, $T_{b}$ is assumed to be the arithmetic mean of the inner and outer boundary temperatures, $\left(T_{i}+T_{o}\right) / 2$, while the average wire temperature is determined from an integral of the temperature profile of the infinitely long fin:

$$
\bar{T}_{w}=\frac{1}{L_{e}} \int_{0}^{L_{e}} T(x) d x, \quad \frac{T(x)-T_{b}}{T_{i}-T_{b}}=e^{-\xi x}
$$

where $L_{e}$ is the effective fin length, determined by solving the temperature distribution equation for the $x$-location where $95 \%$ of the temperature drop has occured:

$$
\frac{T\left(x=L_{e}\right)-T_{b}}{T_{i}-T_{b}}=e^{-\xi L_{e}}=0.05
$$

Solving for the effective fin length gives:

$$
L_{e}=-\frac{1}{\xi} \ln (0.05), \quad \xi=\sqrt{\frac{h_{e f f} P_{w}}{k_{w} A_{w}}}
$$

Substituting and solving for the average wire temperature yields:

$$
\bar{T}_{w}=0.317 T_{i}+0.683 T_{b}
$$

In the case of the thermocouple wires, where two insulated wires are wrapped together with an additional insulation layer, effective wire and insulation diameters and thermal conductivity are used in the preceding calculations, as described by Sparrow (1976):

$d_{w, \text { eff }}=\sqrt{2} d_{w}, \quad d_{i n s, \text { eff }}=\left(\Lambda_{1}+\Lambda_{2}\right) / 2, \quad k_{\text {eff }}=k_{w, 1}+k_{w, 2}$

where $\Lambda_{1}$ and $\Lambda_{2}$ are the cross sectional dimensions of the insulation.

Calculation of the conduction losses through each of the wires and reduction of $Q_{\ell}$ from the results leads to values of $Q$ that are less than those of the pure conduction model, Eq. (11), when the pressure has been sufficiently reduced that the data has reached the diffusive limit and become independent of $R a$. It is assumed that this overprediction of the wire loss is due to the approximations used in the model formulation, including the assumptions of a horizontal circular cylinder geometry and infinite fin length. Due to the complexity of the problem, it may be impossible to formulate a model to accurately predict all conduction losses from the heated body. Therefore, an empirically-derived coefficient, $C_{\ell}$, is introduced to correct the model predictions.

Assuming that the wire loss model correctly accounts for the variations in temperature and gas pressure and provides a maximum value for the total heat loss by conduction, a coefficient having a range of values $0<C_{\ell}<1$ is used to adjust the model as follows:

$$
Q_{\ell}=C_{\ell} * \sum_{i=1}^{N} Q_{\ell, i}
$$


Table 4. CONDUCTION LOSSES MODEL COEFFICIENTS

\begin{tabular}{ccc}
\hline$d_{i}(\mathrm{~mm})$ & $C_{w}$ & $Q_{\ell} / Q$ \\
\hline 80.0 & 0.05 & $1-2 \%$ \\
60.0 & 0.44 & $1-3 \%$ \\
40.0 & 0.20 & $1-3 \%$ \\
25.0 & 0.32 & $3-8 \%$ \\
\hline
\end{tabular}

where $Q_{\ell, i}$ are the model predictions for heat loss from each of the $N$ wires. The value of $C_{\ell}$ for a particular test setting is determined so as to minimize the percent difference between the data and the conduction model, Eq. (11), when the pressure has been sufficiently reduced that the data has reached the diffusive limit. Values of $C_{\ell}$ and the relative portion of the overall heat transfer attributed to conduction losses through the wires are given in Table 4 for each test case. The differences in $C_{\ell}$ values in Table 4 are due to variations in wire length, material and orientation as well as body and heater size.

\section{Test Method}

With the measurement procedure and data reduction techniques defined, the test method is established as follows.

1. Assemble test body in enclosure, fit cold plates, and install completed assembly in vacuum chamber.

2. Seal vacuum chamber and start mechanical and diffusion pumps to establish high vacuum conditions $(K n>100)$.

3. Perform heat capacity and radiation heat transfer tests.

4. Analyze data to obtain $m c_{p}$ and $Q_{r a d}$ correlation.

5. Perform convective heat transfer measurements, starting at atmospheric conditions.

6. Reduce air pressure in chamber and repeat convection measurements, such that at least two tests are performed per decade of $R a_{d_{i}}$ and the data overlaps.

7. Continue reducing pressure and repeating convection tests until diffusive limit is achieved for at least two decades of Rayleigh number.

8. Analyze data to correct for conductive losses.

\section{RESULTS}

Measurements were performed for each of the four inner sphere diameters given in Table 1 according to the test method described in the previous section. The enclosure was maintained at a constant temperature of $22{ }^{\circ} \mathrm{C}$, and the starting and ending values for the temperature difference for the transient convection tests were $50{ }^{\circ} \mathrm{C}$ and $10{ }^{\circ} \mathrm{C}$, respectively. Figure 9 presents all data collected for the $d_{o} / d_{i}=2$ tests, and demonstrates the overlap between data for subsequent tests performed at different

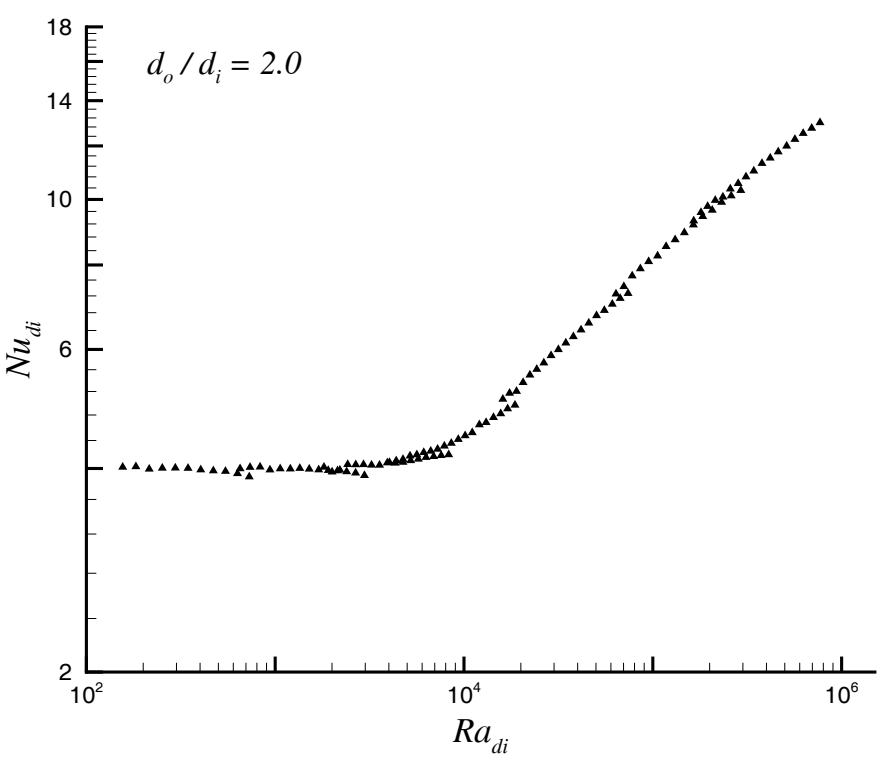

Figure 9. RAW CONVECTION TEST DATA

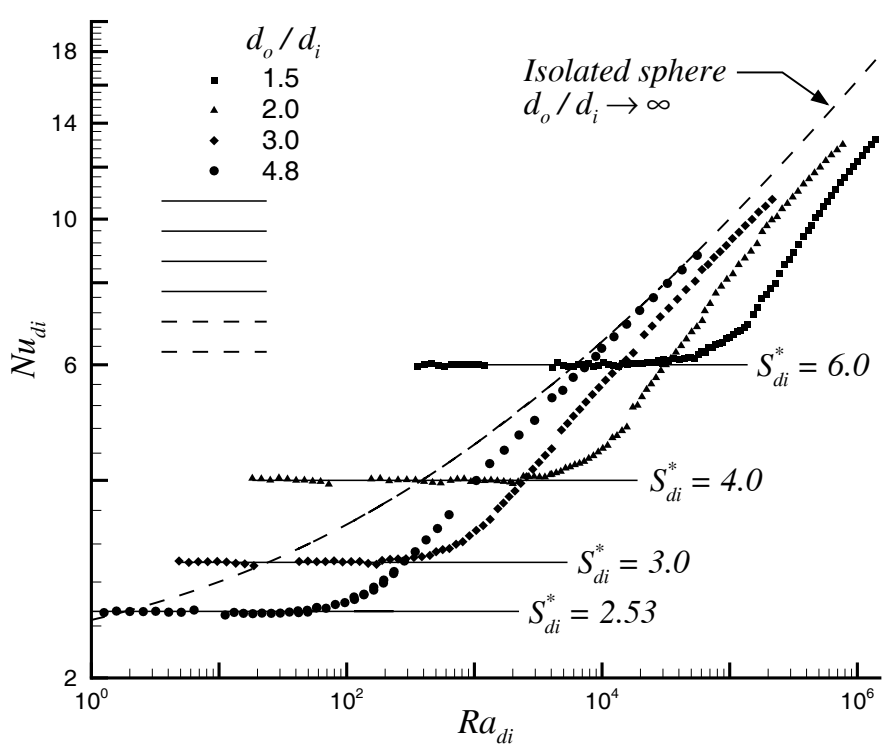

Figure 10. CONVECTION TEST RESULTS

pressure levels. Data are selected from each pressure range to provide a smooth transition and a continuous set of data over the full range of Rayleigh number. The resulting final data sets for each of the four enclosure geometries are plotted in terms of the dimensionless parameters $N u_{d_{i}}$ and $R a_{d_{i}}$ in Figure 10. 


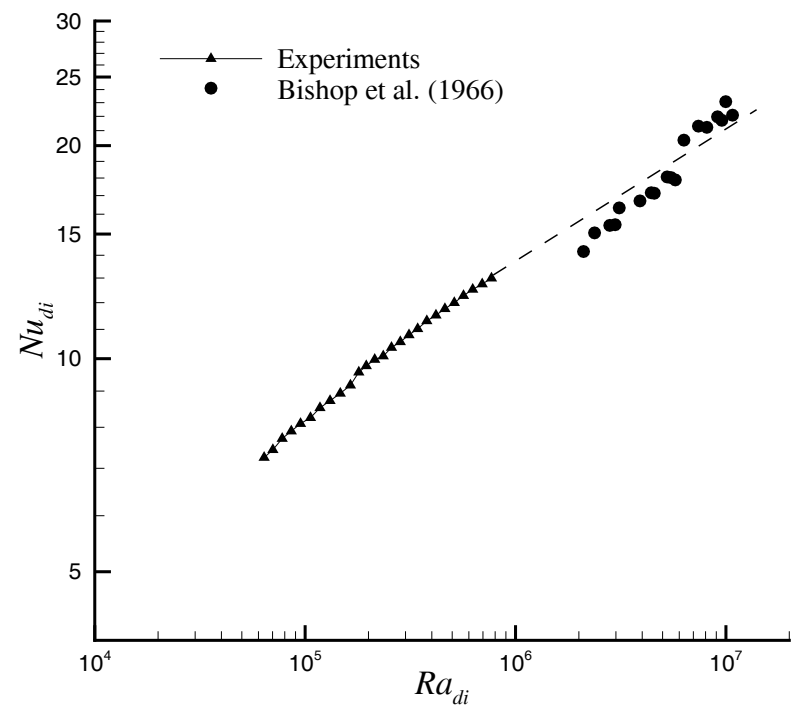

Figure 11. COMPARISON WITH PREVIOUS DATA, $d_{o} / d_{i}=2.0$

A number of observations can be made concerning the data and its trends, as seen in Figure 10. First, the goal of this work, to conduct measurements over a wide range of Rayleigh number, has been achieved with data being generated over at least 4 decades of Rayleigh number in all cases. Second, the data are in excellent agreement with the conductive limit and show independence of $R a_{d_{i}}$ for at least two decades of Rayleigh number. Finally, the data indicate a smooth transition from convection to conduction-dominated heat transfer that occurs within a single decade of the Rayleigh number.

In Figure 10 a model for the isolated, isothermal sphere (Lee et al., 1991) is included, which is equivalent to the limit of an infinitely large enclosure, $d_{o} / d_{i} \rightarrow \infty$

$$
N u_{d_{i}}=S_{d_{i}}^{\star}+F(P r) G_{d_{i}} R a_{d_{i}}^{1 / 4}
$$

where the diffusive limit $S_{d_{i}}^{\star}=2$ and the body gravity function $G_{d_{i}}=0.879$ for the sphere. The value for the Prandtl number function for air at STP is $F(P r)=0.513$. As expected, the isolated sphere model provides an upper bound to the data at the laminar boundary layer flow, high Rayleigh number limit. From Figure 10 it can be seen that, for $d_{o} / d_{i}=4.8$, the dimensions of the enclosure in relation to that of the inner body are large enough such that the system behaves similar to the isolated sphere. As $d_{o} / d_{i}$ decreases, the enclosure walls start to have a larger effect, leading to a reduction in the heat transferred for a given value of $\Delta T$.
Figure 11 compares the experimental data of the present study with the air data of Bishop et al. (1966) for $d_{o} / d_{i}=2$. The Bishop data were measured for larger values of Rayleigh number than were possible in the current test apparatus, so a direct comparison of the data cannot be performed. However, by extrapolating a best fit line from the present data as shown by the dashed line in Figure 11, the good agreement between the measurements and the data of Bishop et al. (1966) can be demonstrated.

\section{SUMMARY}

An experimental procedure and apparatus for performing measurements of natural convection between an isothermal sphere and its surrounding enclosure have been described. The goal of the research project, to provide data over a wide range of Rayleigh number including the transition and diffusive limit, was achieved through the use of a transient test procedure performed in a reduced pressure environment. The proposed transient test method was shown to produce highly accurate data in a much shorter time than the more traditional, steady-state methods. Four different inner spherical bodies were tested and the data were shown to be in excellent agreement with the exact solution for conduction between spherical shells. The data were also compared to existing data from the literature, and were shown to be bounded by the limiting case of natural convection from an isolated, isothermal sphere.

\section{ACKNOWLEDGMENTS}

The authors gratefully acknowledge the Natural Sciences and Engineering Research Council of Canada and Materials and Manufacturing Ontario for their continued financial support of this research.

\section{REFERENCES}

Bishop, E. H., Mack, L. R. and Scanlan, J. A., "Heat Transfer by Natural Convection Between Concentric Spheres," International Journal of Heat and Mass Transfer, Vol. 9, pp. 649 662, 1966.

Scanlan, J. A., Bishop, E. H. and Powe, R. E., "Natural Convection Heat Transfer Between Concentric Spheres," International Journal of Heat and Mass Transfer, Vol. 13, pp. 1857 $1872,1970$.

Weber, N., Powe, R. E., Bishop, E. H. and Scanlan, J. A., "Heat Transfer by Natural Convection Between Vertically Eccentric Spheres," Transactions of the ASME: Journal of Heat Transfer, Vol. 95, pp. 47 - 52, 1973.

Saunders, O. A., "The Effect of Pressure on Natural Convection in Air," Proceedings of the Royal Society: Series A, Vol. 157, pp. 278 - 291, 1936. 
Hollands, K. G. T., "Direct Measurement of Gaseous Natural Convection Heat Fluxes," Proc. First World Conf. Experimental Heat Transfer, Fluid Mechanics and Thermodynamics, eds. R. K. Shah, E. N. Ganic and K. T. Yang, pp. 160 - 168, 1988.

Yovanovich, M. M., "Conduction and Thermal Contact Resistances (Conductances)," Handbook of Heat Transfer, 3rd. ed., eds. W. M. Rohsenow, J. P. Harnett and Y. Cho, McGraw Hill, New York, Chapter 3, pp. 3.1 - 3.73, 1998.

Kennard, E. H., Kinetic Theory of Gases, McGraw Hill, New York, 1938.

Incropera, F. P. and DeWitt, D. P., Fundamentals of Heat and Mass Transfer, 4th ed., Wiley, New York, pp. 96 - 99, 114 118, 1996.

Raithby, G.D. and Hollands, K.G.T., "Natural Convection," Handbook of Heat Transfer, 4th ed., eds. W.M. Rohsenow, J.P Hartnett and Y. Cho, McGraw Hill, NY, Chapter 4, 1998.

Sparrow, E. M., "Error Estimates in Temperature Measurement," Measurements in Heat Transfer, 2nd ed., eds. E. R. G. Eckert and R. J. Goldstein, Hemisphere, Washington, pp. 3 - 6, 1976.

Lee, S., Yovanovich, M. M. and Jafarpur, K., "Effects of Geometry and Orientation on Laminar Natural Convection Heat Transfer from Isothermal Bodies," Journal of Thermophysics and Heat Transfer, Vol. 5, pp. 208 - 216, 1991. 